\title{
Diffractive production of Drell-Yan pairs and heavy flavors
}

\author{
Boris Kopeliovich \\ Max-Planck-Institut für Kernphysik, Postfach 30980, 69029 Heidelberg, Germany \\ \& Joint Institute for Nuclear Research, 141980 Moscow Region, Dubna, Russia
}

\begin{abstract}
Partonic interpretation of high-energy reactions is known to depend on a reference frame. Particularly, in the rest frame of the target Drell-Yan process looks like a freeing of the projectile $l \bar{l}$ fluctuation, rather than $q \bar{q} \rightarrow l \bar{l}$ annihilation. The lightcone representation for Drell-Yan reaction is very similar to that in DIS and exposes a substantial contamination of soft interactions which turns out to be dominant in diffractive production of lepton pairs and in nuclear shadowing. We estimate the fraction of diffractive events in the total Drell-Yan cross section, which scales in $M^{2}$, and find a substantial deviation from factorization. An analogous approach is developed for diffractive production of heavy flavors.
\end{abstract}

\section{Light-cone representation for Drell-Yan reaction}

Drell-Yan mechanism of lepton pair production in hadronic collisions at small $x_{2} \ll 1$ has much in common with deep-inelastic lepton scattering at small Bjorken $x_{B j}$ [1]-[3] (we use the standard notations [4] $x_{1}-x_{2}=x_{F}$ and $x_{1} x_{2}=M^{2} / s$, where $x_{F}$ and $M$ are the Feynman variable and the effective mass of the lepton pair). Actually, the factorization theorem is a reflection of such a similarity. However, for our purpose (diffraction, nuclear shadowing) it is convenient to use the light-cone representation for Drell-Yan process [1]-[3]. In the rest frame of the target proton the projectile beam hadron is surrounded by a parton cloud which contains with a small probability a lepton pair. The freeing of the leptons by means of interaction between the projectile partons and the target is equivalent to the Drell-Yan mechanism [4] of lepton pair production.

The cross section of lepton pair production can be represented in a factorized form [1]-[3]

$$
M^{2} \frac{d \sigma_{D Y}^{h N}}{d M^{2} d x_{1}}=\int_{x_{1}}^{1} d \alpha\left(\frac{x_{1}}{\alpha}\right) \Phi_{q}^{h}\left(\frac{x_{1}}{\alpha}\right) \int d^{2} r_{T} W_{q l \bar{l}}\left(\alpha, r_{T}\right) \sigma(q \rightarrow q l \bar{l})
$$


Here $M$ is the effective mass of the lepton pair. $\Phi_{q}^{h}(x)$ is the quark distribution function in the projectile hadron. $W_{q l \bar{l}}\left(\alpha, r_{T}\right)$ is the light-cone distribution function for the qli Fock component of the projectile quark in the mixed $r_{T}-\alpha$ representation, where $r_{T}$ is the transverse separation between the quark and the center of gravity of the $l \bar{l}$-pair, and $\alpha$ is the fraction of the light-cone momentum of the projectile quark carried by the $l \bar{l}$ pair. The distribution over $r_{T}$ has a form [1, 2]

$$
W_{q l \bar{l}}\left(\alpha, r_{T}\right) \propto \kappa^{2} K_{1}^{2}\left(\kappa r_{T}\right)
$$

where

$$
\kappa^{2}=(1-\alpha) M^{2}+\alpha^{2} m_{q}^{2} .
$$

Here $m_{q}$ is the effective quark mass which preserves the fluctuation from too large transverse separations compared to the confinement radius. We take into account only the transversely polarized component of the virtual photon, since the contribution of the longitudinally polarized one to diffraction or nuclear shadowing is a higher twist effect.

There is a close similarity between the distribution functions for a $q \bar{q}$ fluctuation of a virtual photon [5] and Eq. (2) . This is because the energy denominators corresponding to fluctuations $\gamma^{*} \rightarrow \bar{q} q$ and $q \rightarrow q \bar{l} l$ are very similar.

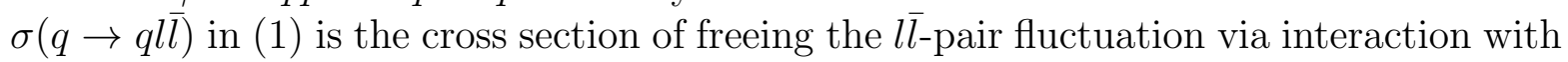
the target. Surprisingly, it turns out to be equal to the total interaction cross section with a nucleon of a colorless $q \bar{q}$ pair with a transverse separation $\alpha r_{T}$,

$$
\sigma(q \rightarrow q l \bar{l})=\sigma_{\bar{q} q}\left(\alpha r_{T}, x_{2}\right)
$$

We included here a dependence on $x_{2}$ of the gluon density in the target. In Born approximation the dipole cross section is independent of $x_{2}$ and reads [6]

$$
\sigma_{\bar{q} q}\left(r_{T}\right)=\frac{16}{3} \int \frac{d^{2} q \alpha_{s}^{2}}{\left(q^{2}+m_{g}^{2}\right)^{2}}\left(1-e^{i \vec{q} \vec{r}_{T}}\right)\left[1-F_{N}(q)\right] .
$$

Here $\vec{q}$ is the transverse momentum of the exchanged gluons, $m_{g}$ is the effective gluon mass which takes care of confinement, $F(q)=\left\langle N\left|\exp \left[i \vec{q}\left(\vec{r}_{1}-\vec{r}_{2}\right)\right]\right| N\right\rangle$ is the two-quark formfactor of the nucleon, where $\vec{r}_{1,2}$ are the transverse coordinates of the quarks coupled to the gluons.

Expression (5) is infrared stable even if $m_{g}=0$ due to color screening, as different from the quark-nucleon total cross section which is divergent in this case. We should emphasize that $\sigma(q \rightarrow q l \bar{l})$ is not an interaction cross section of a single colored quark, but a production cross section. The former is infra-red divergent, but the latter is finite. Indeed, a fluctuation can be produced on mass shell only if the interaction amplitude of the projectile Fock states with and without the fluctuation are different, otherwise the coherence of the projectile is not disturbed.

Eq. (1) can also be interpreted in terms of Feynman diagrams [1, 2] shown in Fig. 11. The $\bar{l} l$ pair production is due to interactions with the target which occur prior and after the radiation. The impact parameters of the quark before and after radiation pair are different by a distance $\alpha r_{T}$. The corresponding color screening factor turns out to be exactly the same as in the total cross section of a colorless $\bar{q} q$ pair having transverse separation $\alpha r_{T}$ [1, 2]. 


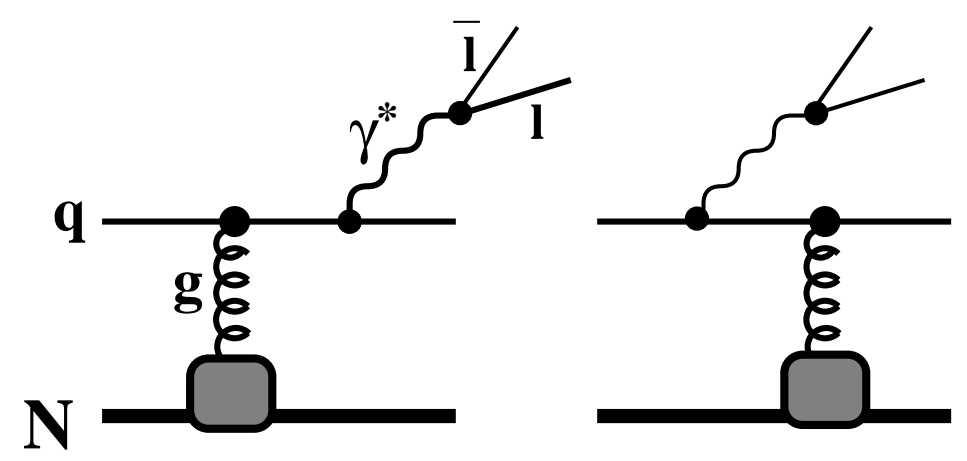

Figure 1: Feynman diagrams for lepton pair production. The grey boxes represent the gluon distribution function for the proton.

Naively, one would expect that a typical transverse separation for a heavy $l \bar{l}$ fluctuation is of the order of $1 / M^{2}$. This, however, might not be true, it depends on $\alpha$. According to (2) - (3) the mean transverse separation squared is (see also in [7])

$$
\left\langle r_{T}^{2}\right\rangle \approx \frac{1}{(1-\alpha) M^{2}}
$$

Thus, rare asymmetric $l \bar{l}$ fluctuations with $1-\alpha \sim \mu^{2} / M^{2}$, where $\mu$ is a hadronic mass scale, interact softly. This is why the soft contribution to the $l \bar{l}$ production cross section is not small and scales in $M^{2}$ (compare with DIS [9]). This is illustrated in Table 1 which represents the $\bar{q} q$ dipole cross section $\langle\sigma\rangle$ and $\left\langle\sigma^{2}\right\rangle$ averaged over different fluctuations weighted by the distribution function $W$ as one can read in (四).

Table 1. Contributions of soft and hard $l \bar{l}$ fluctuations of a quark to the inclusive and diffractive cross sections of Drell-Yan reaction.

\begin{tabular}{|c|l|l|l|l|}
\hline Fluctuation & $W_{q l \bar{l}}$ & $\sigma$ & $W_{q l \bar{l}} \sigma$ & $W_{q l \bar{l}} \sigma^{2}$ \\
\hline Hard & $\sim 1$ & $\sim 1 / M^{2}$ & $\sim 1 / M^{2}$ & $\sim 1 / M^{4}$ \\
\hline Soft & $\sim \mu^{2} / M^{2}$ & $\sim 1 / \mu^{2}$ & $\sim 1 / M^{2}$ & $\sim 1 / \mu^{2} M^{2}$ \\
\hline
\end{tabular}

We classify conventionally, for the sake of simplicity, all the fluctuations to be soft or hard. The Table shows that soft ones have the same $M^{2}$ dependence since the rareness of their appearance is compensated by the large cross section. This is a manifestation of the aligned jet model of Bjorken and Kogut [8] for Drell-Yan lepton pair production.

\section{Nuclear shadowing}

This gives a hint to calculation of nuclear shadowing for $l \bar{l}$ production. In the lowest order for multiple scattering expansion it has a form [9],

$$
\frac{\sigma(q A \rightarrow l \bar{l} X)}{A \sigma(q N \rightarrow l \bar{l} X)}=1-\frac{1}{4} \frac{\left\langle\sigma^{2}\right\rangle}{\langle\sigma\rangle}\langle T\rangle F_{A}^{2}\left(q_{L}\right)
$$


The mean value of the dipole cross section is defined as

$$
\langle\sigma\rangle=\int_{0}^{1} d \alpha \int d^{2} r_{T} W_{q l \bar{l}}\left(\alpha, r_{T}\right) \sigma_{\bar{q} q}\left(\alpha \cdot r_{T}\right)
$$

The mean value $\left\langle\sigma^{2}\right\rangle$ is dominated by soft fluctuations according to Table 1 . It has the same $1 / M^{2}$ dependence as the total cross section $\langle\sigma\rangle$, therefore nuclear shadowing scales in $M^{2}$.

In (7) the mean nuclear thickness function $\langle T\rangle$ and the longitudinal nuclear formfactor are defined as,

$$
\langle T\rangle=\frac{1}{A} \int d^{2} b T^{2}(b)
$$

where

$$
\begin{gathered}
T(b)=\int_{-\infty}^{\infty} d z \rho_{A}(b, z) \\
F_{A}^{2}\left(q_{L}\right)=\frac{1}{A\langle T\rangle} \int d^{2} b\left|\int_{-\infty}^{\infty} d z e^{i q_{L} z} \rho_{A}(b, z)\right|^{2} .
\end{gathered}
$$

The longitudinal momentum transfer $q_{L}$ depends on the final $q \bar{l}$ effective mass and approximately equals to $q_{L} \approx 2 m_{N} x_{2}$ (see, however, $\| 10$. For heavy pairs $M>4 G e V$ in the experiments at SPS CERN at $p_{l a b}=200 \mathrm{GeV}$ the value of $q_{L}$ is pretty large and the formfactor suppresses nuclear shadowing. An onset of nuclear effects was observed for the first time in the experiment E772 at $800 \mathrm{GeV} \mathrm{[11]} \mathrm{at} \mathrm{Fermilab} \mathrm{in} \mathrm{a} \mathrm{good} \mathrm{agreement} \mathrm{with}$ formula (17) [1, 2, 12].

At much higher energies of RHIC and LHC one reaches the regime $q_{L} \ll 1 / R_{A}$ and the formfactor $F_{A}^{2}\left(q_{L}\right)=1$. Then the lifetime of the $l \bar{l}$ fluctuation exceeds the nuclear radius. In this case one can easily sum up all the higher multiple scattering terms by a simple replacement in (11),

$$
\sigma(q \rightarrow q l \bar{l}) \Rightarrow 2\left[1-\exp \left(-\frac{1}{2} \sigma(q \rightarrow q l \bar{l}) T(b)\right)\right]
$$

Since nuclear shadowing for Drell-Yan reaction is essentially eliminated by the nuclear formfactor in (ㅍ) in the energy range of SPS, this reaction can be safely used for normalization of nuclear suppression for $J / \Psi$ production in heavy collisions as function of centrality. However, at higher energies, particularly at RHIC, the longitudinal momentum transfer

$q_{L} \approx 2 m_{N} M^{2} / s$ in (11) vanishes and Eq. (12) exposing a full strength of nuclear shadowing is valid. Nuclear suppression for Drell-Yan reaction becomes quite strong (a factor of 0.5 or less for collision of heavy nuclei) what makes normalization for nuclear suppression of $J / \Psi$ problematic.

\section{Diffraction}

Nuclear shadowing discussed above is known to have close relation to diffraction [13]. Feynman diagrams for diffractive production of a lepton pair in quark-nucleon interaction are depicted in Fig. 2. One needs two gluons in the amplitude to insure a large rapidity gap, 

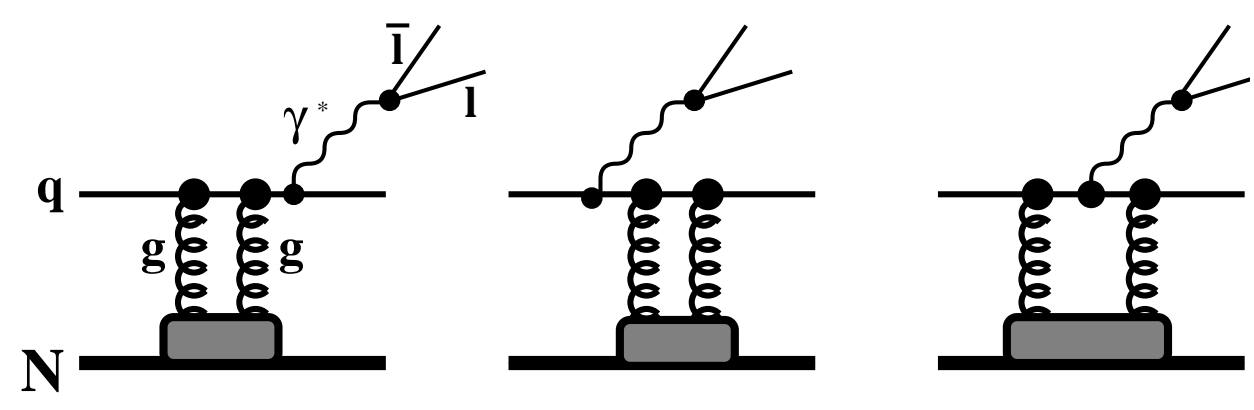

Figure 2: Feynman diagrams for diffractive production of lepton pair. The grey boxes represent the double-gluon distribution function for the proton.

which corresponds to an experimental definition of diffraction. Therefore, the diffractive cross section is related by usual expression to the dipole cross section,

$$
\left.\int d M^{2} \frac{d \sigma_{d d}^{D Y}}{d M^{2} d p_{T}^{2}}\right|_{p_{T}=0}=\frac{\left\langle\widetilde{\sigma}^{2}\right\rangle}{16 \pi}
$$

with only modification marked by the tilde. Namely, one of the exchanged gluons can be attached to another projectile spectator parton (one of the two gluons still must couple the radiating quark [14]). As a result, additional color screening suppresses the cross section $\tilde{\sigma}_{\bar{q} q}\left(r_{T}, x_{2}\right)$ as compared to the conventional $\bar{q} q$-nucleon dipole cross section $\sigma_{\bar{q} q}\left(r_{T}, x_{2}\right)$

$$
\tilde{\sigma}_{\bar{q} q}\left(r_{T}\right)=\frac{16}{3} \int \frac{d^{2} q \alpha_{s}^{2}}{\left(q^{2}+m_{g}^{2}\right)^{2}}\left(1-e^{i \vec{q}_{T}}\right)\left[1-F_{N}(q)\right]\left[\mathbf{1}-\boldsymbol{F}_{\boldsymbol{h}}(\boldsymbol{q})\right],
$$

Where $F_{h}(q)$ is the double-quark formfactor of the hadron defined in the same way as $F_{N}(q)$. We use here again Born approximation, for the sake of clarity, in analogy to (5). However, this expression differs from Eq. (5) by the last factor highlighted in bold, which takes into account the extra color screening of the Drell-Yan process by the spectator partons. This is not a big effect for small $r_{T}$ since large $q$ suppresses the projectile hadron formfactor $F_{h}(q)$. However, diffraction, as was demonstrated above, is dominated by soft interactions, i.e. by large $r_{T}$ of about the size of the hadron. Therefore, the last factor in (14) substantially suppresses the share of diffraction in the total cross section compared to that in DIS, known to be about $10 \%$. Using (14) and (5) and assuming the same $t$-slopes in both diffractive reactions we get,

$$
\left(\frac{\sigma_{d d}}{\sigma_{t o t}}\right)^{D Y} \approx \frac{1}{2}\left(\frac{\sigma_{d d}}{\sigma_{t o t}}\right)^{D I S}
$$

The calculation is done for a proton beam. The difference between DIS and Drell-Yan reaction is a direct manifestation of violation of factorization in diffraction. There is also another source of deviation from factorization which brings an additional suppression to the 
diffraction cross section. This is the survival probability factor. There is an extra condition in diffractive Drell-Yan reaction for the spectator partons in the projectile hadron to have no inelastic interaction. This can be roughly estimated in eikonal approximation like it was done in [15]. The result depends on energy and ranges from few percent at the Tevatron collider energy up to about $20 \%$ at lower energies.

\section{Heavy flavors}

The light-cone dynamics of heavy flavor production is very similar to that for Drell-Yan reaction, except the pair of heavy quarks can be also radiated by the t-channel gluon (see Feynman diagrams in [14]) and it can participate in the interaction with the target. The impact parameter representation for the cross section has a form similar to (11) [1, 16],

$$
M^{2} \frac{d \sigma_{\bar{Q} Q}^{h N}}{d M^{2} d x_{1}}=\int_{x_{1}}^{1} d \alpha\left(\frac{x_{1}}{\alpha}\right) \Phi_{q}^{h}\left(\frac{x_{1}}{\alpha}\right) \int d^{2} r_{T} W_{q \bar{Q} Q}\left(\alpha, r_{T}\right) \sigma(q \rightarrow q \bar{Q} Q) .
$$

We use the same notations as in (1). The distribution function $W_{q \bar{Q} Q}\left(\alpha, r_{T}\right)$ for a $q \bar{Q} Q$ fluctuation has the same form as $W_{q \bar{l} l}\left(\alpha, r_{T}\right)$, except replacement $\alpha_{e m}^{2} \rightarrow 2 \alpha_{s}^{2} / 3$. The cross section of $\bar{Q} Q$ production in $q-N$ interaction turns out to be equal to the color dipole cross section for interaction of a colorless system $\bar{q} q g^{*}$ with a nucleon (compare with (仿) $\sigma(q \rightarrow \bar{Q} Q q)=\sigma_{\bar{q} q g^{*}}\left(\alpha, r_{T}\right)$ [1, 16] which has a form [17,

$$
\sigma_{\bar{q} q g^{*}}\left(\alpha, r_{T}\right)=\frac{8}{9}\left[\sigma_{\bar{q} q}\left((1-\alpha) r_{T}\right)+\sigma_{\bar{q} q}\left(r_{T}\right)\right]-\frac{1}{8} \sigma_{\bar{q} q}\left(\alpha r_{T}\right)
$$

Here the virtual gluon $g^{*}$ represents the color-octet $\bar{Q} Q$ pair. We neglect the spatial separation in the $\bar{Q} Q$ because it is small and the corresponding correction to the diffractive cross section considered in 18 is suppressed by factor $1 / M_{\bar{Q} Q}^{2}$ (see Table 1$)$.

To calculate diffractive production of heavy flavors one should perform a replacement analogous to (17) for $\widetilde{\sigma}\left(\alpha r_{T}\right)$ in (13) - (15). The cross section of diffractive production of heavy quarks is subject to the same strong suppression by the survival probability factor as was discussed above for Drell-Yan reaction.

Comparison with available data needs to take into account the exclusive channels and the kinematical domain where the diffractive production was observed. This will be done in a separate publication.

Acknowledgements This work was partially supported by European Network: Hadronic Physics with Electromagnetic Probes, No FMRX CT96-0008.

\section{References}

[1] B.Z. Kopeliovich, in proceedings of the Workshop Hirschegg'95: Dynamical Properties of Hadrons in Nuclear Matter, ed. by H. Feldmeier and W. Nörenberg, Darmstadt, 1995, p. 102 ( hep-ph/9609385)

[2] O. Benhar, B.Z. Kopeliovich and A. Zieminski, (1993), unpublished 
[3] S.J. Brodsky, A. Hebecker and E. Quack, Phys.Rev. D55 (1997) 2584

[4] S.D. Drell and T.M. Yan, Phys. Rev. Lett., 25 (1970) 316

[5] J.M. Bjorken, J.B. Kogut and D.E. Soper, Phys. Rev. D3 (1971) 1386;

N.N. Nikolaev and B.G. Zakharov, Z. Phys. C49 (1991) 607

[6] Al.B. Zamolodchikov, B.Z. Kopeliovich and L.I. Lapidus, Sov. Phys. JETP Lett. 33 (1981) 612

[7] S.J. Brodsky, P. Hoyer, A.H. Müller and W.-K.Tang, Nucl. Phys. B 369 (1992) 519

[8] J.D. Bjorken and J. Kogut, Phys. Rev. D8, (1973) 1341

[9] B. Kopeliovich and B. Povh, Phys.Lett. B367 (1996) 329; Z.Phys. A356 (1997) 467

[10] B.Z. Kopeliovich, J. Raufeisen and A.V. Tarasov, hep-ph/9807211, to appear in Phys. Lett. B

[11] D.M. Alde et al, Phys. Rev. Lett. 64 (1990) 2479.

[12] B.Z. Kopeliovich, J. Raufeisen, A. Schäfer and A.V. Tarasov, paper in preparation

[13] V.N. Gribov, Sov. Phys. JETP 29 (1969) 483

[14] J.F. Gunion and G. Bertsch, Phys. Rev. D25 (1982) 746

[15] E. Gotsman, E. Levin and U. Maor, Phys. Lett. B438 (1998) 229

[16] B.Z. Kopeliovich, A. Schäfer and A.V. Tarasov, hep-ph/9808378

[17] N.N. Nikolaev and B.G.Zakharov, JETP 78 (1994) 598

[18] N.N. Nikolaev, G. Piller and B.G.Zakharov, Z. Phys. A354 (1996) 99 